\title{
GPPS-BJ-2019-0166
}

\section{Very-Large Eddy Simulation of complex turbulent flame based on FGM}

\author{
Xingsi Han \\ College of Energy and Power Engineering, \\ Nanjing University of Aeronautics and \\ Astronautics \\ xshan@nuaa.edu.cn \\ Nanjing, 210016, China
}

\author{
Pengxiang Wan \\ College of Energy and Power Engineering, \\ Nanjing University of Aeronautics and \\ Astronautics \\ 18018065442@163.com \\ Nanjing, 210016, China
}

\author{
Junkui Mao \\ College of Energy and Power Engineering, \\ Nanjing University of Aeronautics and \\ Astronautics \\ Corresponding author: mjkpe@nuaa.edu.cn \\ Nanjing, 210016, China
}

\begin{abstract}
High fidelity simulation of complex turbulent flame is still challenging from many aspects. The present study aims to make the progress from two aspects. The first one is the turbulence modeling, which is achieved by using the newly developed VLES (Very-Large Eddy Simulation) method. The VLES method is a self-adaptive turbulence modeling method which can resolve the turbulence based on the local mesh resolution and the local turbulence scale information. It evolves smoothly from RANS to DNS with the increase of the mesh resolution. It thus can provide accurate predictions on relatively coarse meshes. The second one is the combustion modeling, which is done by combining the FGM (Flamelet Generated Manifold) method. FGM method is an efficient chemistry reduction technique, which has attracted attentions for the successful applications to various complex combustion systems. The present study thus makes the effort to combine the VLES and FGM methods to model complex turbulent flames. Two test cases are selected, i.e. the classical Sandia D flame and the swirling premixed flame from a single GE LM6000 combustor. The numerical results are compared with available experiments. It is found that the VLES-FGM method can be successfully applied for the high fidelity simulation of the complex test flames. The VLES method has high potentials for turbulent flame simulations.
\end{abstract}

\section{INTRODUCTION}

High fidelity simulation of turbulent combustion is a hot research topic for decades. However, due to the complex interactions of turbulence and combustion in the process, it is not easy to obtain the accurate predictions of turbulent combustion. Large eddy simulation of turbulent combustion has been applied successfully and shown the potentials for high fidelity simulation (Rochette et al., 2018). However, the huge computational cost makes it very difficult to apply LES for wide application of turbulent combustion.

There are several methods which have been proposed to reduce the huge computational cost of LES for combustion problem. In the aspect of turbulence modelling, unified turbulence modelling has developed rapidly in recent years. The unified turbulence modelling means that the turbulence is modelled based on the local gird resolution and the local turbulence scales, which can make the turbulence modelling process to evolve smoothly from RANS, LES to DNS. Thus, the coarse mesh can be used for high Reynolds number turbulent flow and also the accuracy can be maintained. In recent years, VLES (Very-Large Eddy Simulation) (Han and Krajnovic, 2013; Han and Krajnovic, 2015) has been found to be an efficient modelling approach for complex turbulent flow simulations. It has been validated in many classical turbulent flows. However, VLES method for turbulent combustion problem is few studied. It is thus quite straightforward to extend the high potential VLES modelling method for complex turbulent combustion simulations. It is supposed that the VLES based turbulent combustion simulation can use coarse mesh to obtain accurate predictions. However, the validation is quite few in previous literatures. It is thus the first goal for the present study, i.e. apply VLES for turbulent combustion simulation. 
The second goal of the present study comes from the turbulence combustion modelling. Combustion is a very complex chemical reaction process. Detailed chemistry mechanism is desired in order to gain more details during the combustion. However, the direct calculation of the detailed chemistry needs huge computational costs which makes it nearly impossible for most of combustion simulations, especially when LES method is applied. The possibility to resolve the reaction zone in a typical LES computational cell coupled with the detailed chemistry information provided by tabulated strategies, like the FGM method (Flamelet Generated Manifold) (Oijen and Goey, 2002), makes this combustion modelling technique attractive in order to capture the complex turbulent flame structures. The FGM has been found to be an efficient method to modelling turbulent combustion (Benim et al., 2019). The FGM modelling method assumes that the scalar evolution in a turbulent flame can be approximated by the scalar evolution in a laminar flame by a few key variables, such as the mixture-fraction, scalar-dissipation and reaction-progress, etc. The computational cost of detailed combustion is thus significantly reduced by using FGM.

The combination of VLES turbulence modelling and the FGM combustion modelling method is thus attractive for complex turbulent combustion simulations. The second goal of the present study is thus to validate the performance of the combined VLES-FGM method.

To this end, two selected turbulent flames are adopted for the present simulation method. The first one is the piloted jet flame, namely Sandia Flame D. The second one is the swirling premixed flame which comes from a single GE LM6000 combustor. The two cases are widely studied in previous literatures, which make them good test cases for the present study of VLES-FGM method.

\section{NUMERICAL METHODOLOGY}

For the VLES turbulence modelling, the present study applies the $\mathrm{k}-\varepsilon$ turbulence model as the underlying method. By introducing the resolution control function, Fr, the VLES method can be achieved, and the function, Fr, is the core of VLES modelling. It has the form as shown in Eq. (1), where $L_{\mathrm{c}}, L_{\mathrm{i}}$, and $L_{\mathrm{k}}$ are the cut-off length scale, the integral length scale and the Kolmogorov length scale, respectively. The three scales represent the mesh resolution scale, the largest scale and the smallest scale, respectively.

$F r=\min \left[1.0,\left(\frac{1.0-\exp \left(-\beta L_{\mathrm{c}} / L_{\mathrm{k}}\right)}{1.0-\exp \left(-\beta L_{\mathrm{i}} / L_{\mathrm{k}}\right)}\right)^{2}\right]$

The Fr has a value between 0 and 1.0. Depending on the local scale information, it can evolve within this range and model the turbulence with difference levels. Thus, it can smoothly evolve from RANS to DNS.

The governing equations for the VLES modelling are shown in Eqs. (2) - (4). The turbulence stress is reformulated with the introduction of Fr. More details about the VLES modelling approach and its implementation can be found in other references (Han and Krajnovic, 2013; Han and Krajnovic, 2015).

$$
\begin{aligned}
& \mathrm{D} \bar{\rho} k / \mathrm{D} t \\
& =P_{k}-\bar{\rho} \varepsilon+\partial\left[\left(\mu+\mu_{\mathrm{t}} / \sigma_{\mathrm{k}}\right) \partial k / \partial x_{j}\right] / \partial x_{j} \\
& \mathrm{D} \bar{\rho} \varepsilon / D t \\
& =(\varepsilon / k)\left(C_{\varepsilon 1} P_{k}\right. \\
& \left.-C_{\varepsilon 2} \bar{\rho} \varepsilon\right)+\partial\left[\left(\mu+\mu_{\mathrm{t}} / \sigma_{\varepsilon}\right) \partial k / \partial x_{j}\right] / \partial x_{j} \\
& \mu_{\mathrm{t}}=F r \cdot \bar{\rho} C_{\mu} k^{2} / \varepsilon
\end{aligned}
$$

For the combustion modelling, the present study applies the FGM approach with the assumed PDF (beta-PDF). The detailed chemical reaction mechanism can be considered, and the computational cost of detailed combustion calculation is significantly reduced at the same time. Here, the partially premixed FGM method is used. In the simulations, the mixture fraction $\widetilde{Z}$ and its variance $\widetilde{Z^{\prime \prime 2}}$, the progress variable $\tilde{C}$ and its variance $\widetilde{C^{\prime \prime}}{ }^{2}$, are calculated with the following governing equations (5) - (8).

$$
\begin{aligned}
& \mathrm{D} \bar{\rho} \tilde{Z} / D t=\partial\left[\left(\lambda / C_{\mathrm{p}}+\mu_{\mathrm{t}} / S c_{\mathrm{t}}\right) \partial \tilde{Z} / \partial x_{j}\right] / \partial x_{j} \\
& \mathrm{D} \bar{\rho} \widetilde{Z^{\prime \prime}} / D t=\partial\left[\left(\lambda / C_{\mathrm{p}}+\mu_{\mathrm{t}} / S c_{\mathrm{t}}\right) \partial \widetilde{Z^{\prime \prime}} / \partial x_{j}\right] / \partial x_{j}+ \\
& C_{\mathrm{g}} \mu_{\mathrm{t}}\left(\partial \tilde{Z} / \partial x_{j}\right)^{2}-C_{\mathrm{d}} \bar{\rho}(\varepsilon / k) \widetilde{Z^{\prime \prime}} \\
& \mathrm{D} \bar{\rho} \tilde{C} / D t=\partial\left[\left(\lambda / C_{\mathrm{p}}+\mu_{\mathrm{t}} / S c_{\mathrm{t}}\right) \partial \tilde{C} / \partial x_{j}\right] / \partial x_{j}+\bar{\rho} \widetilde{\tilde{\omega}_{\mathrm{C}}} \\
& \mathrm{D} \bar{\rho} \widetilde{C^{\prime \prime}} / D t=\partial\left[\left(\lambda / C_{\mathrm{p}}+\mu_{\mathrm{t}} / S c_{\mathrm{t}}\right) \partial \widetilde{C^{\prime \prime}} / \partial x_{j}\right] / \partial x_{j}+ \\
& c_{\phi}\left(\mu_{\mathrm{t}} / S c_{\mathrm{t}}\right)\left(\partial \tilde{C} / \partial x_{j}\right)^{2}-\left(\bar{\rho} c_{\phi} / \tau_{\mathrm{turb}}\right) \widetilde{C^{\prime \prime}}
\end{aligned}
$$

The present numerical simulation is conducted using a general purpose commercial software and the present VLES modelling is implemented in the software via external subroutine. For the combustion simulation, the widely applied GRI 3.0 mechanism is used for the methane and air reaction. For the numerical schemes, the convective terms are discretized with the second order central differencing scheme coupled with a small fraction of upwind scheme. Central differencing scheme is applied for the diffusion terms. As for the temporal term, it is approximated with the second order implicit scheme.
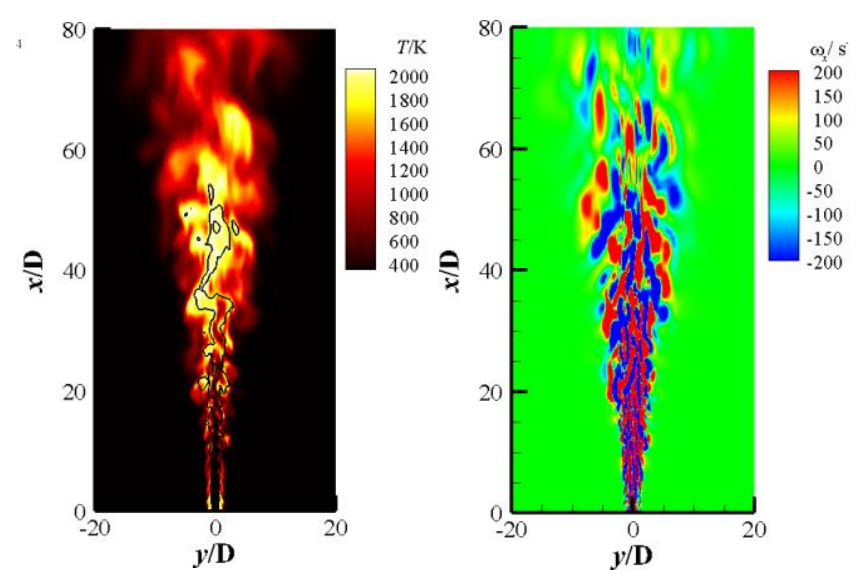

Figure 1 Instantaneous contour of temperature and the vorticity by VLES-FGM for Sandia flame D. 


\section{PILOTED JET FLAME - SANDIA FLAME D}

Sandia Flame D is a widely studied test case for various high fidelity numerical simulations (Pitsch and Steiner, 2000). It is a piloted coaxial methane air flame. The fuel is a mixture of $25 \%$ methane and $75 \%$ air. The bulk velocity at the fuel inlet is $49.6 \mathrm{~m} / \mathrm{s}$. The pilot velocity is $11.4 \mathrm{~m} / \mathrm{s}$ and the co-flow air has a velocity of $0.9 \mathrm{~m} / \mathrm{s}$. The Reynolds number is around 22400 based on the fuel inlet velocity and the diameter of the jet $\mathrm{D}(\mathrm{D}=7.2 \mathrm{~mm})$.

The computational grid is $215 \times 140 \times 64$, resulting in a total cells about 1.92 million. The grid is generated referring to other ref. (Vreman et al., 2008). Local mesh refinement is carried out in the jet shear layer and the central flame area. The results of the fully developed circular pipe calculated by RANS are used as the velocity, turbulent kinetic energy and turbulent dissipation rate distribution at the inlet. Also, the turbulence fluctuations at the fuel inlet and pilot inlet are generated by synthetic vortex method. The time step is set to a fixed value of $5 \times 10^{-5} \mathrm{~s}$.
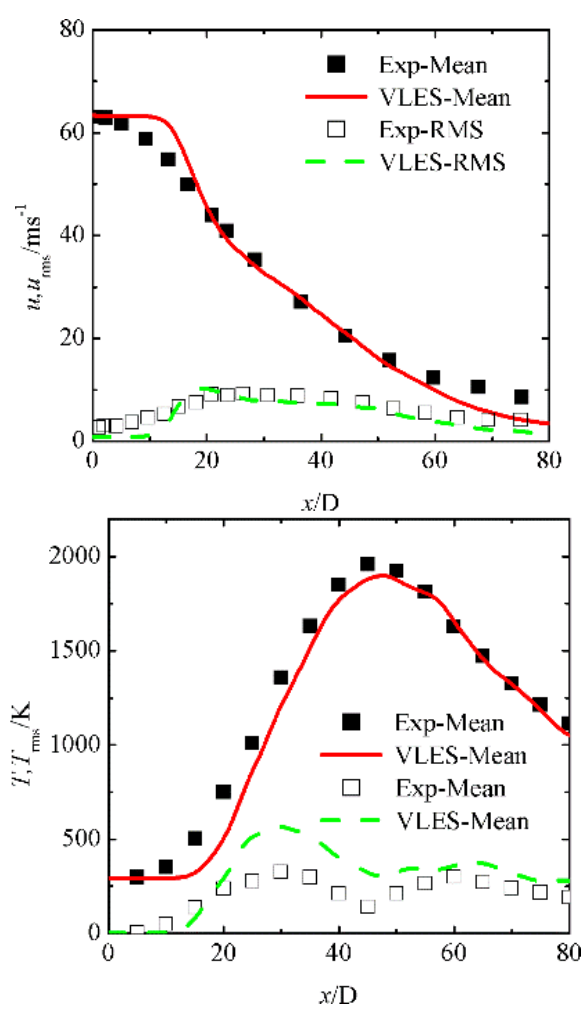

Figure 2 Axial profiles of mean and RMS of velocity and temperature by VLES-FGM for Sandia flame D.

Figure 1 shows the instantaneous results of the predicted temperature and the vorticity for Sandia Flame D by the present VLES-FGM method. It can be seen that the highly unsteady fluctuation is resolved by the present numerical method. It can be seen from the instantaneous vortex contour that the strong turbulence motion mainly occurs in the central jet area, which expands gradually from the jet inlet to the downstream region, and the scale of the vortex increases gradually. The main area of combustion reaction can be found by the instantaneous temperature contour, in which the area of $x / D>20$ is the high temperature reaction zone. In that region, the fuel and oxygen are mixed well, the chemical reaction and turbulence motion are strong. In the area of $x / D$ $<20$, the local turbulence scale near the fuel outlet is very small, and the mixing caused by the small scale turbulence is weak and the reaction is relatively weak.

The time-averaged results of the velocity and the temperature are given in Figure 2, with experimental data. It demonstrates that the numerical predictions agree well with the experimental data, which confirms the suitability and accuracy of the present numerical method for the piloted jet flame. It should be noted that the differences between the prediction and the experimental data mainly locate in the inlet region. One possible reason for the difference is the inaccurate inlet boundary conditions in the present numerical simulation. It also shows the importance of inlet turbulence for the downstream flow development of jet mixing. Based on previous publications, there are many other factors which may result in the difference such as the chemistry tabulation method, the turbulence combustion model, turbulence model, radiation, etc.
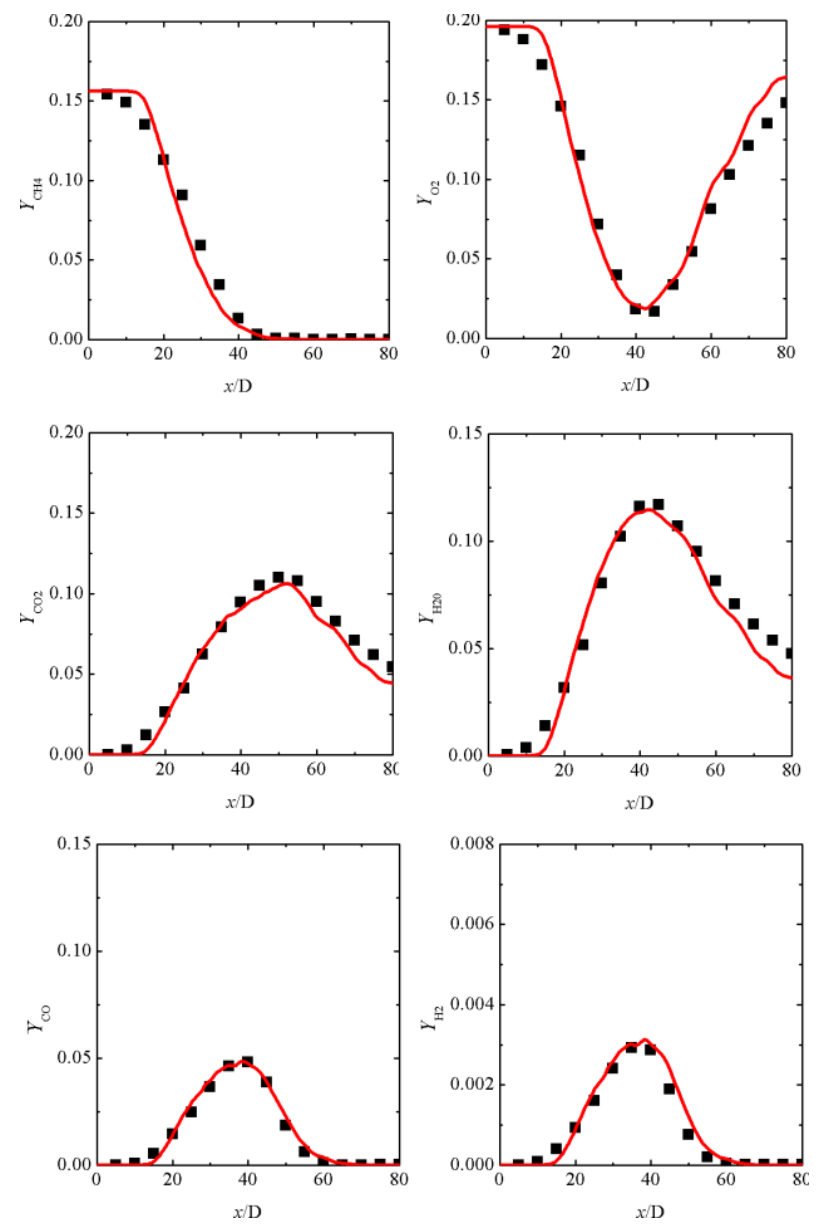

Figure 3 Axial profiles of mean mass fraction of main species. Symbols: experimental data; solid line: VLES-FGM predictions.

Figure 3 shows the distribution of the time averaged mass fraction along the axis for several main species. By comparing with the experimental results, it can be found that the prediction of the average value of the main components by VLES-FGM is in good agreement with the experimental 
values. The local deviation from the experimental results is mainly close to the boundary at the inlet. This shows that the VLES-FGM method can predict the distribution of main reaction species accurately.

Figure 4 show the contours of the mean and RMS velocity and temperature, respectively. The mean velocity demonstrates a typical jet flow structures, including the jet core and the downstream developed regions. From the RMS velocity results, it can be seen that the main turbulence fluctuations locate at the boundaries between the central net and the piloted jet. The mean and RMS temperature results show that the main combustion occurs at the downstream region of the jet and the fluctuations of the temperature have two main regions, the one around the central jet and the one at the edge of the piloted flame around the central jet. The results demonstrate that the present VLES-FGM method can give quite good prediction of the whole flow fields.
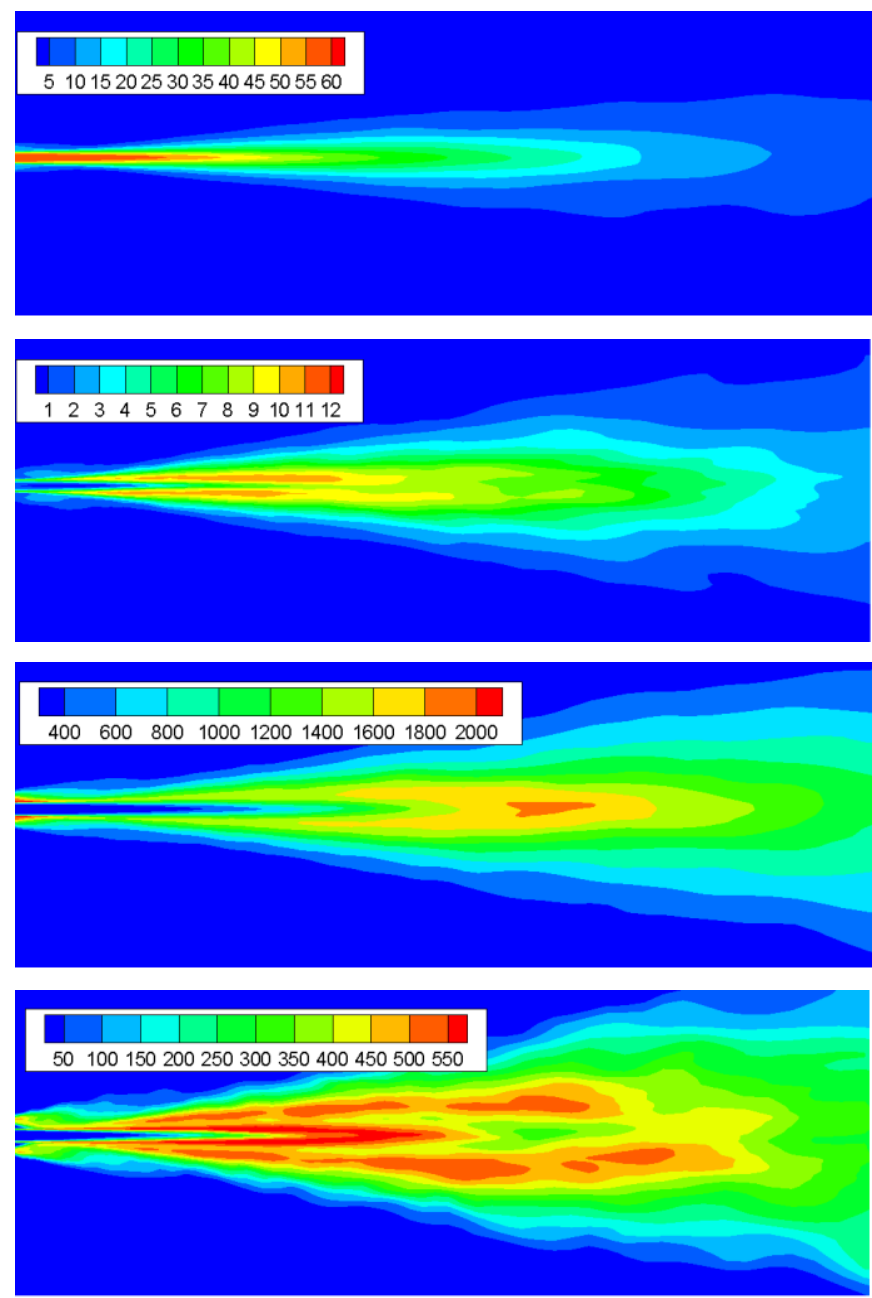

Figure 4 Contours of the mean velocity, RMS velocity, mean temperature, RMS temperature of the Sandia D flame by the VLES-FGM method.

\section{PREMIXED FLAME - GE LM6000 COMBUSTOR}

The second test case is a more complex, engineering related flame which corresponds to the swirling premixed combustion in a model combustor. The model combustor is taken from the GE's lean premixed dry low NOx emissions
LM6000 gas turbine combustor. A highly swirling jet is injected from a circular inlet under high pressure $\left(\mathrm{P}_{0}=6.18 \mathrm{E} 5\right.$ $\mathrm{Pa})$ and high temperature $\left(\mathrm{T}_{0}=644 \mathrm{~K}\right)$ conditions. The selected combustor has a relatively simple geometry, which has a rectangular box with two slots at the bottom and top from which the cooling air is injected.

The inlet is the premixed mixture of methane and air with the equivalence ratio of 0.56 (corresponding to a lean premixed methane-air mixture), which results in the flame temperature of around $1810 \mathrm{~K}$. The swirling number is around 0.56. Based on previous studies of swirling flows, this swirl number corresponds to the regime where the onset of a vortex breakdown happens. The Reynolds number at the inlet is around 320000 based on the inlet bulk velocity and the diameter of the inlet pipe $(D=34 \mathrm{~mm})$ for premixed mixture. The Reynolds number is high enough such that the fully developed turbulence assumption is reasonable for the present numerical study.

Structured computational mesh is applied in the present case, and the final computation grid contains about 2.1 million cells. At the inlet, the inflow velocity profiles (axial, radial and azimuthal) are applied from previous study (Fureby, 2010) which ensures the accurate inlet velocity boundary condition for the VLES calculations. It should be noted that, in the present study and the experiments, the two side boundaries are solid walls, which means that the present swirling flame is confined in a rectangular box.
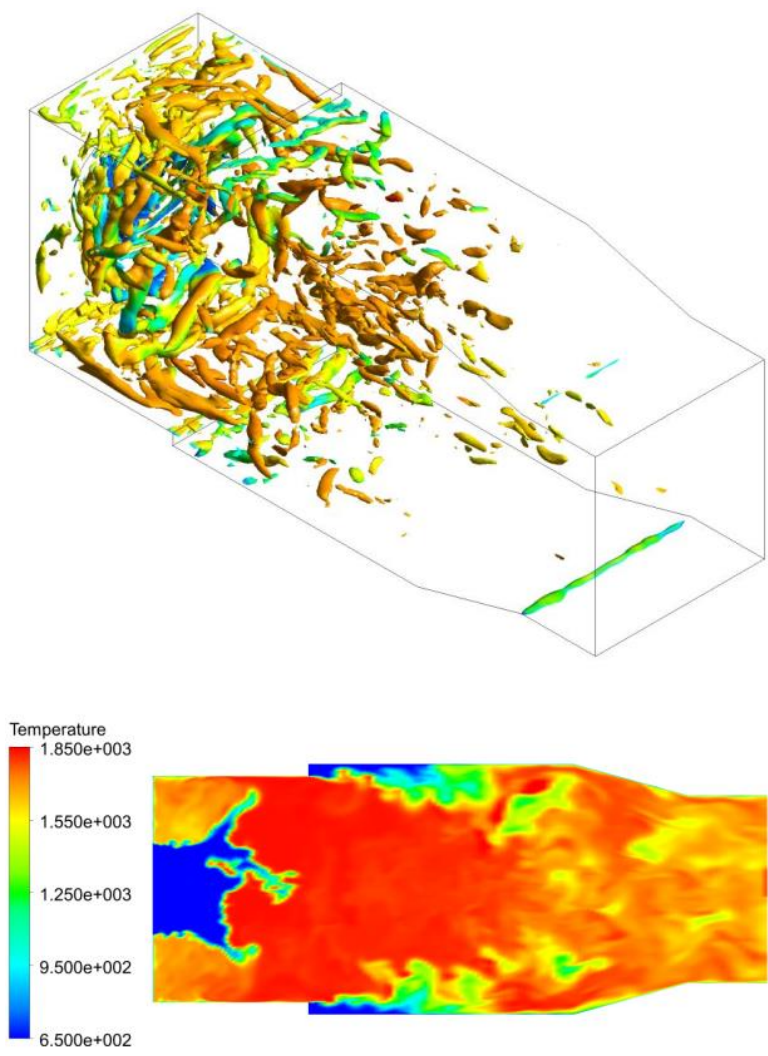

Figure 5 Instantaneous contours of the $Q$ turbulence structure and the temperature by VLESFGM for GE premixed combustor. 

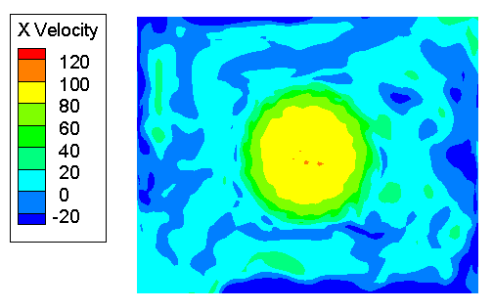

\begin{tabular}{|c|}
\hline Temperature \\
$\square 1800$ \\
1650 \\
1500 \\
1350 \\
1200 \\
1050 \\
900 \\
750 \\
600 \\
\hline
\end{tabular}

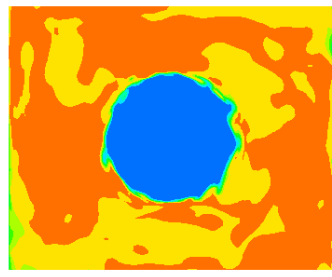

(a)

(b)
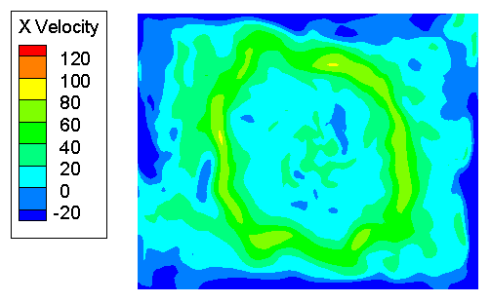

(c)
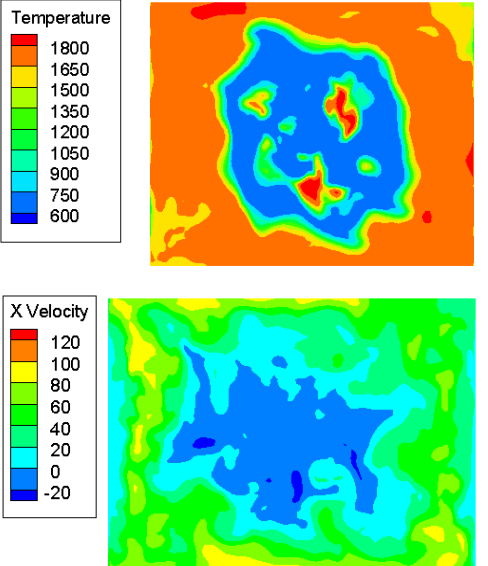

(d)

(e)

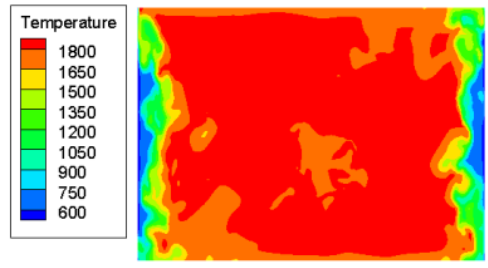

(f)

Figure 6 Instantaneous contours of the axial velocity and the temperature at three locations: (ab) $x / D=0.18$; (c-d) $x / D=0.72$; (e-f) $x / D=2.33$ by VLESFGM for GE premixed combustor.

Figure 5 shows the instantaneous turbulence structures by the $Q$ values and the instantaneous temperature in a central plane by the present VLES-FGM method for the premixed combustion. The unsteady premixed swirling flame can be seen clearly. Figure 6 give the instantaneous contours of the axial velocity and the temperature at three downstream locations of $\mathrm{x} / \mathrm{D}=0.18,0.72$ and 2.33 respectively. The swirling incoming premixed jet expands rapidly and results in a forward stagnation point. All the figures show the strong unsteadiness, and the high swirl flow demonstrates a very complex vortex shedding pattern with significant azimuthal flow structures. With the strong swirling characteristics of the incoming flow, the shear layers are quickly breakdown when traveling downstream.

The results in Figure 6 (especially Figures 6(c) and 6(e)) also show that, with the expanding of the swirling jet, there exist complex interactions of the vortex and the neighboring walls. Secondary vortex can be seen clearly at the corners of the walls. It means that the wall confinement has significant effects on the swirling flow.
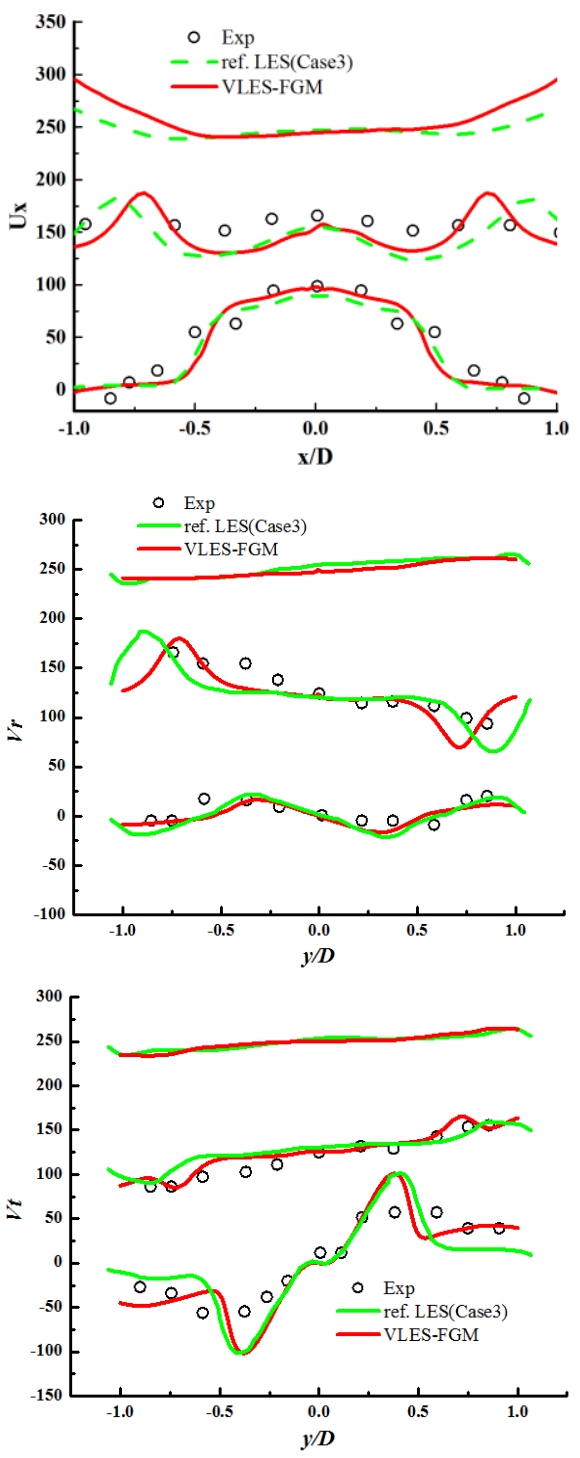

Figure 7 Comparisons of the mean axial, radial and tangential velocity by the present VLES-FGM method, previous LES and experiments at three downstream locations $(x / D=0.18,0.72$ and 2.33 ) for

GE premixed combustor. The profiles at $x / D=$ 0.72 and 2.33 are shifted $125 \mathrm{~m} / \mathrm{s}$ and $250 \mathrm{~m} / \mathrm{s}$, respectively.

Figure 7 shows the comparisons of the present VLESFGM predictions of the mean axial, radial and tangential 
velocities with previous LES and available experiments, at three downstream locations. It can be seen that the present VLES-FGM method gives quite good predictions compared with the experimental data (Hura et al., 1998), and previous LES results (Fureby, 2010). The results also confirm the suitability and accuracy of the present VLES-FGM numerical method for complex swirling premixed flame in engineering related combustor.

The results in Figure 7 show that the present VLESFGM gives the shear layer locations slightly closer to the central region of the combustor compared with previous LES studies. There are still some differences between the present VLES and the experiments. One possible reason may be that the swirling premixers used in the experiments are neglected in the present simulations, while the swirling jets are used at the inlet to represent the swirling flow. That is, the swirling premix dynamics may be important for the simulations. The results can be seen clearly of the tangential velocity distributions at the location of $\mathrm{x} / \mathrm{D}=0.18$ (which is very close to the flow inlet). Although the profiles of the inlet velocity are used at the inlet, the velocity at slightly downstream location cannot be well predicted by the previous LES and the present VLES. It demonstrates that the geometry of the complex swirlers should be considered in the swirling flow simulations.

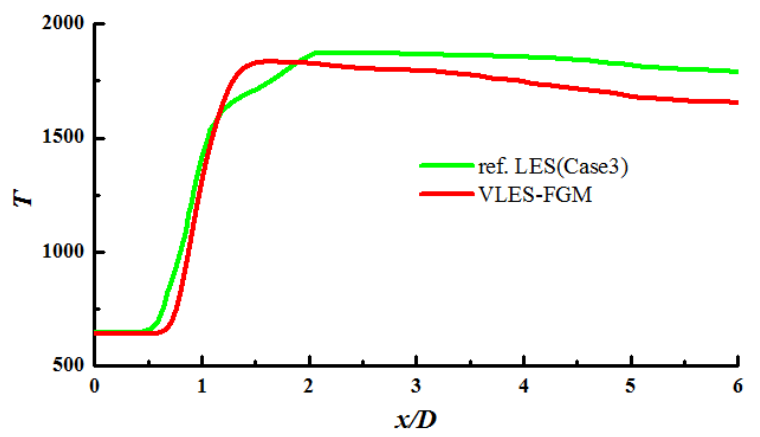

\section{Figure 8 Comparisons of the mean temperature by the present VLES-FGM method and previous LES along the central axial line for GE premixed combustor.}

Figure 8 shows the mean temperature at the central line between the present VLES-FGM method and previous LES. It can be seen that the results are generally agree with each other well. The temperature starts to increase rapidly at the location of around $\mathrm{x} / \mathrm{D}=0.7$ from the present VLES-FGM method. It is slightly closer to the inlet from previous LES. It should be noted that there is no information about the up and down cooling jets and the lower temperature of the present VLES method at the downstream location may result from larger cooling velocity given by the present VLES than previous LES studies.

\section{CONCLUSIONS}

The present study applies the newly developed VLES turbulence modelling method and the FGM combustion modelling method for two different complex turbulent flames, i.e. the piloted jet flame (Sandia Flame D) and the swirling premixed flame from a GE LM6000 combustor. It is found that the VLES-FGM method by the present study can give quite good predictions using relatively coarse mesh. The unsteady turbulence and combustion process can be resolved reasonably. The results also demonstrate the potentials of the VLES modelling method for complex turbulent flow and turbulent combustion simulations.

\section{References}

Benim A.C., Pfeiffelmann B., Ocłoń P., et al. (2019). Computational investigation of a lifted hydrogen flame with LES and FGM. Energy 173, 1172-1181.

Fureby C. (2010). LES of a multi-burner annular gas turbine combustor. Flow, Turbulence and Combustion 84, 543-564.

Han X. and Krajnovic S. (2013). An efficient very large eddy simulation model for simulation of turbulent flow. International Journal for Numerical Methods in Fluids 71 (11), 1341-1360.

Han X. and Krajnovic S. (2015). Very-Large Eddy Simulation Based on k- $\omega$ Model. AIAA Journal 53, 11031108.

Hura H. S., Joshi N. D., Mongia H. C., and Tonouchi J. (1998). Dry low emission premixer CCD modelling and validation. ASME 98-GT-444.

Oijen J., Goey L. H. (2002). Modelling of premixed counterflow flames using the flamelet-generated manifold method. Combustion Theory \& Modelling 6, 463-478.

Pitsch H., Steiner H. (2000). Large-eddy simulation of a turbulent piloted methane/air diffusion flame (Sandia flame D). Physics of Fluids 12(10), 2541-2554.

Rochette B., Collin-Bastiani F., Gicquel L., et al. (2018). Influence of chemical schemes, numerical method and dynamic turbulent combustion modeling on LES of premixed turbulent flames. Combustion and Flame 191, 417-430.

Vreman A.W., Albrecht B.A., Oijen J.A.V, et al. (2008). Premixed and nonpremixed generated manifolds in largeeddy simulation of Sandia flame D and F. Combustion \& Flame 153, 394-416.

\section{ACKNOWLEDGMENTS}

This work was financially supported by the National Natural Science Foundation of China (grant Nos: 51606095 and 91841302), the Jiangsu Provincial Natural Science Foundation (grant No: BK20160794), and the Aerospace Power Foundation of China. X.S. Han acknowledges the support of the Jiangsu Specially-Appointed Professor Program. 